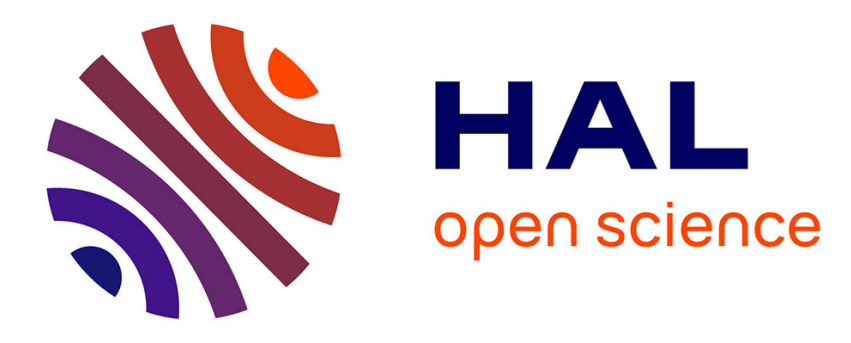

\title{
Multiaxial experiments with radial loading paths on a polymeric foam
}

Adrien Donnard, Sandra Guerard, Laurent Maheo, Philippe Viot, Gérard Rio

\section{To cite this version:}

Adrien Donnard, Sandra Guerard, Laurent Maheo, Philippe Viot, Gérard Rio. Multiaxial experiments with radial loading paths on a polymeric foam. Polymer Testing, 2018, 67, pp.441-449. hal-01785073

\section{HAL Id: hal-01785073 \\ https://hal.science/hal-01785073}

Submitted on 4 May 2018

HAL is a multi-disciplinary open access archive for the deposit and dissemination of scientific research documents, whether they are published or not. The documents may come from teaching and research institutions in France or abroad, or from public or private research centers.
L'archive ouverte pluridisciplinaire HAL, est destinée au dépôt et à la diffusion de documents scientifiques de niveau recherche, publiés ou non, émanant des établissements d'enseignement et de recherche français ou étrangers, des laboratoires publics ou privés. 


\title{
Multiaxial experiments with radial loading paths on a polymeric foam
}

\author{
Adrien Donnard ${ }^{\mathrm{a}}$, Sandra Guérard ${ }^{\mathrm{a}}$, Laurent Maheo ${ }^{\mathrm{b}, \mathrm{c}, *}$, Philippe Viot $^{\mathrm{a}}$, Gérard Rio $^{\mathrm{b}}$ \\ a Arts et Metiers ParisTech, I2M, CNRS UMR 5295, F-33405 Talence, France \\ ${ }^{\mathrm{b}}$ Univ Bretagne Sud, IRDL, CNRS UMR 6027, F-56321 Lorient, France \\ ' St-Cyr Military Academy, CREC, F-56381 Guer, France
}

\section{A R T I C L E I N F O}

\section{Keywords:}

Cellular materials

Multiaxial experiments

Analyzing shape and volume changes

\begin{abstract}
A B S T R A C T
Cellular materials such as polymeric foams in particular have been widely studied under uniaxial loading conditions. Many experimental studies have been focusing recently, however, on the responses of these foams to multiaxial loads. In the present study, a novel experimental hexapod device was used to perform combined uniaxial compression and simple shear tests. Using a post-processing method of analysis which can be used to study elementary mechanical behavior, the authors show the occurrence of non-proportional stress paths in the material under investigation although proportional kinematic paths were imposed. A failure limit criterion is presented for use with the foam of interest. The results of the present analysis yield useful information for meeting our future objective, namely to develop a numerical model for simulating multiaxial loading conditions.
\end{abstract}

\section{Introduction}

Cellular materials have been widely used for many years for manufacturing safety equipment and protective applications. Because of their considerable dissipative capacity and their weak force transmission properties, they are good candidates for protecting both goods and persons. The choice of cellular materials, which range from soft polymer foam to rigid aluminum foam, depends on the damping power required. Mills et al. [1], for instance, have studied the use of polymer foams for producing several types of personal protection, such as cushions, shoes and helmets. Fernandes and Alves de Sousa [2] recently published a review focusing on motorcycle helmets and showed the importance of including polymeric foam liners in the helmet design. The authors of the previous study [2] have also briefly addressed the topic of Finite Element models, citing several studies in which impact tests were simulated with a view to optimizing helmet design. In the present context of engineering applications, Yang and Shim [3] have recommended using a macroscopic description of the responses observed, taking elastomer foams to constitute a homogeneous continuum. Foam behavior can therefore be decomposed into elementary behavior such as hyperelasticity, viscosity and irreversible transformations.

When drawing up numerical models simulating foams subjected to mechanical loads, it is necessary to characterize the material. In most previous studies, foams have been characterized experimentally by performing uniaxial compression tests, which are easy to apply. The influence of the strain rate or the impact speed has often been studied to account for the normal conditions of use, as in Refs. [4-7]. However, the validity of these characterizations and models is questionable when the mechanical loads are no longer uniaxial loads. Some models such as Ogden's model [8] have taken several loading conditions into account. This model, which was developed for rubber-like materials, has been extended to compressible materials by including the changes of volume in addition to the shape change behavior. This distinction is a natural way of describing the hyperelastic behavior of rubber-like and cellular materials, and has been adopted in several models such as the MooneyRivlin $[9,10]$ model, which is based on tensor invariants, and the Ogden model, which is based on principal stretches.

Several authors have naturally performed multiaxial experiments to provide numerical models with data. Volume change behavior has been studied by performing hydrostatic tests [4,11-14] and shape change behavior, by performing simple shear tests $[4,15,16]$. In both cases, the question of finding suitable methods for the post-processing analysis multiaxial data arises. In the case of large transformations, Criscione et al. [17] used an invariant basis for natural strains consisting of the amount-of-dilatation, the magnitude-of-distortion and the mode-of-distortion. These authors recommended using natural or Hencky strain and Cauchy stress tensors to study and compare the results of multiaxial loading tests. Combaz et al. $[18,19]$ recently used these post-processing tools to analyze the results of tests on aluminum and polymeric foams subjected to multiaxial loads.

In the present study, quasi-static multiaxial experiments were performed with radial loading paths on a polymeric foam. This Polypropylene foam has been studied in detail by Viot et al. $[5,7,12]$ by

\footnotetext{
* Corresponding author. Univ Bretagne Sud, IRDL, CNRS UMR 6027, F-56321 Lorient, France.

E-mail address: laurent.maheo@univ-ubs.fr (L. Maheo).
} 


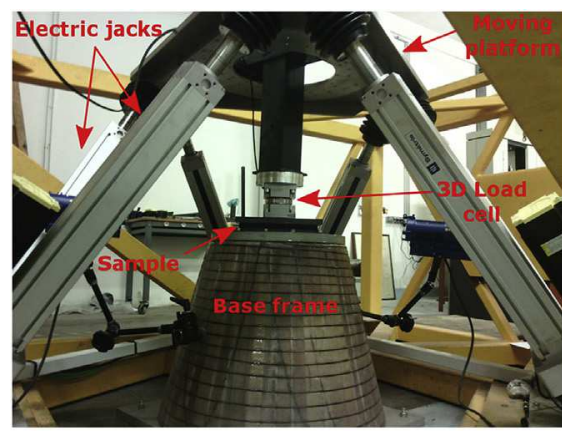

(a) Hexapod in its environment.

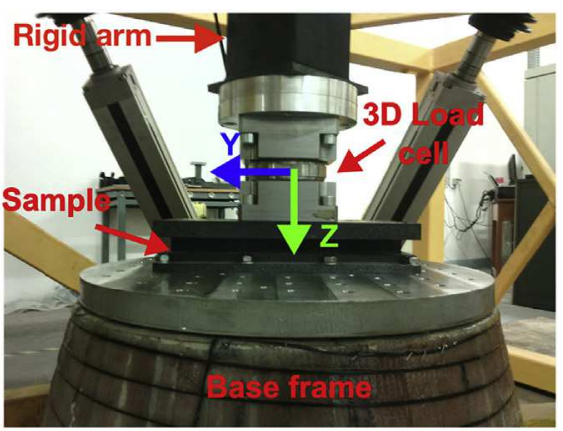

(b) Zoom on the sample.

Fig. 1. The hexapod device. A black $300 \times 20 \times 20 \mathrm{~mm}$ sample of EPP foam was glued between two steel plates.

performing uniaxial and hydrostatic compression tests at several strain rates. In the present study, simple shear tests and tests involving simple shear combined with uniaxial compression were conducted using a novel hexapod device [20] with which controlled shape changes and volume changes can be imposed. Using the method of analysis presented in Refs. [18,19], shape change and volume change processes were analyzed and compared between various imposed radial loading paths.

In the first part of this study (Section 2), the mechanical behavior of the Expanded Polypropylene foam under investigation is briefly described and the size of the specimens used is explained. The hexapod device and the post-processing method of analysis used are also presented. In the second part (Section 3), the results of basic experiments such as uniaxial compression and tension and simple shear tests are presented. The multiaxial loading experiments performed are described and the results are presented and discussed in Section 4. Lastly, the main results obtained are summarized in section 5 .

\section{Material and methods}

\subsection{Material}

\subsubsection{Polypropylene foam}

This study was performed on a closed-cell expanded polypropylene (EPP) foam called Arpro supplied by the company JSP. This foam has been widely studied by the present authors, who have established that the strain rate and the density both affect the mechanical behavior of the material under uniaxial compression [5] and hydrostatic compression [12] loading conditions. To extend this database, a new specimen of the same material with a mean density of $85.3 \mathrm{~kg} \mathrm{~m}^{-3}$ (standard deviation $4.3 \mathrm{~kg} \mathrm{~m}^{-3}$ ) was prepared.

\subsubsection{Description of the samples}

Two main characteristics have to be taken into consideration when performing mechanical tests. First, the specimen size has to be suitable for obtaining representative, fairly homogeneous elementary mechanical responses. Secondly, the shape of the specimen must correspond to the types of loading applied in the tests.

In the case of the present uniaxial tension tests, a dogbone specimen designed in line with standard NF EN ISO 1798 [21] was used in order to concentrate the strain in the thinnest part of the specimen.

In the case of simple shear tests, the shape of the specimen greatly affects the results [22,23]. Simple shear tests induce a tangential force and a normal force on the loaded faces. These two forces combined induce a compression load and a tensile load near the free edges [24,25], which could be regarded as a bending load. This process can be reduced by increasing the elongation ratio $\frac{l}{h}$ of the specimen, where $l$ and $h$ are the length and the height of the specimen, respectively.
Bouvier et al. [25], for instance, used an elongation ratio of 10 on a metal sheet sample, G'Sell et al. [24] recommended a ratio of more than 15 in the case of polymers, and Mostafa et al. [26] used a ratio of 13 in that of a foam sample. Specimens measuring $300 \mathrm{~mm}$ long, $20 \mathrm{~mm}$ high and $20 \mathrm{~mm}$ wide, having an aspect ratio $\frac{l}{h}$ of 15 were chosen here for performing both simple shear and uniaxial compression tests.

\subsection{Methods}

\subsubsection{Experimental devices}

A classical electromechanical Zwick Z250 Roell device was used to perform quasi-static uniaxial tensile tests in line with the procedure defined in the French standard NF EN ISO 1798 [21]. A crosshead speed of $41.25 \mathrm{~mm} \mathrm{~min}^{-1}$ was imposed in order to obtain a strain rate of $0.0125 \mathrm{~s}^{-1}$. Uniaxial stress was obtained using a $10 \mathrm{kN}$ sensor and strain field with Digital Image Correlation (D.I.C.) VIC2D software from pictures recorded by a camera (CANON EOS 50D) at a frequency of $1 \mathrm{~Hz}$.

Multiaxial experiments tests were performed using a hexapod facility. Fig. 1 shows the hexapod, which is a modified Gough-Stewart platform, a type of parallel robot constituted of a fixed and a moving platform. Thanks to 6 electromechanical jacks, the top plate can be moved independently in the six degrees of freedom, corresponding to three translation axes and three rotation axes. Its horizontal velocity can reach $1.4 \mathrm{~m} / \mathrm{s}$ and the maximal vertical velocity is $1 \mathrm{~m} / \mathrm{s}$. For the multiaxial tests, a rigid arm is mounted perpendicular to the moving top platform. Specimens were glued between 2 steel plates, one of which was screwed onto a rigid arm and the other, onto a base frame. This arrangement made it possible to apply loads of all kinds to the sample, including even complex loads such as combinations of movements. The hexapod was able to reach speeds of up to $1 \mathrm{~m} / \mathrm{s}$ in all directions. However, the present study focused simply on quasi-static loading performed at a speed of $0.25 \mathrm{~mm} / \mathrm{s}$. Strain rates of $\dot{\varepsilon}_{z z}=0.0125 \mathrm{~s}^{-1}$ and $\dot{\gamma}_{y z}=0.0125 \mathrm{~s}^{-1}$ were imposed at each test so as to be able to compare the results obtained without any involvement of the viscous contribution.

Forces were obtained using a piezoelectric 3D sensor (Kistler 9377C) mounted between the rigid arm and the sample. The local basis $(\mathrm{X}, \mathrm{Y}, \mathrm{Z})$ of the sensor was defined as shown in Fig. $1 \mathrm{~b}$. The sensor was set at 2, 10, and $10 \mathrm{kN}$ in the $\mathrm{X}, \mathrm{Y}$ and $\mathrm{Z}$ directions, respectively. Electrical signals were then amplified and recorded at a frequency of $100 \mathrm{~Hz}$, as can be seen in Fig. 2 in the particular case of a uniaxial compression applied on the Z-axis and a simple shear applied simultaneously on the YZ-plane.

As in the tensile tests, the displacements and strain fields were calculated using the D.I.C. technique. The region of interest in the D.I.C. was chosen so as to rule out the occurrence of boundary effects by excluding the areas on both sides of the specimen and those near the steel plates (see Fig. 3). A second camera was placed perpendicularly to 


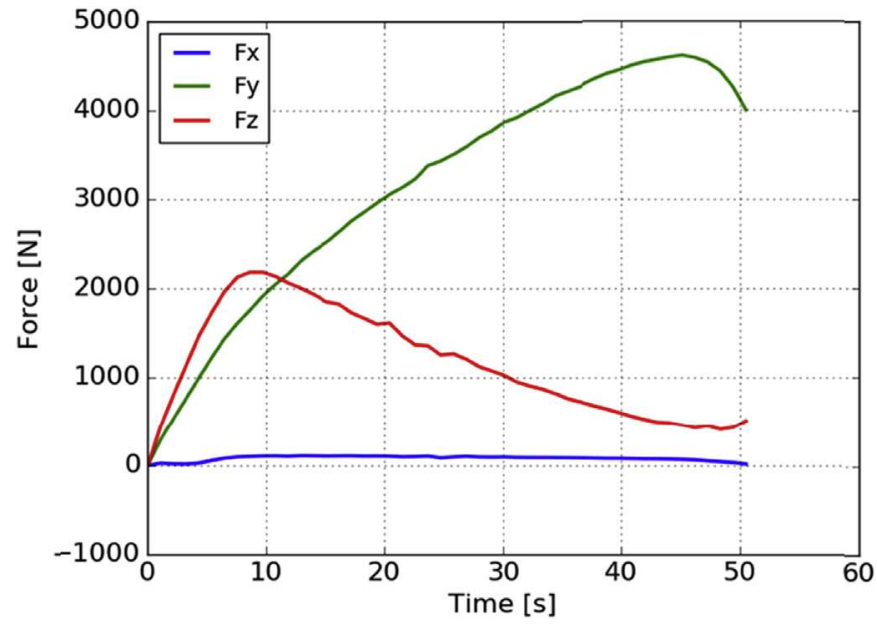

Fig. 2. Forces recorded under simultaneous uniaxial compression and simple shear loads.

the first one in order to detect any transversal strains occurring during the application of uniaxial compression and simple shear loads.

\subsubsection{Post-processing of the multiaxial test data}

In the case of a simple shear load or a uniaxial compression load or both loads applied simultaneously, the components of the symmetric Cauchy stress tensor expressed in the hexapod coordinate system can be written as in equation (1).

$\left[\sigma_{i j}\right]=\left[\begin{array}{ccc}0 & 0 & 0 \\ 0 & 0 & \sigma_{y z} \\ 0 & \sigma_{y z} & \sigma_{z z}\end{array}\right]$ with $\sigma_{z z}=\frac{F_{z}}{S}$ and $\sigma_{y z}=\frac{F_{y}}{S}$

where $F i$ is the force recorded in the $i$-direction, and $S$ is the current $z$ normal section. It is important to note that in both experiments, although the shear components $\sigma x y$ and $\sigma x z$ were not measured, they were assumed to be null because EPP foam is generally reputed to be macroscopically homogeneous. The $F x$ force component recorded by the sensor was almost equal to 0 . Even if it means that the average global value of $\sigma_{x x}$ is vanishingly small, the local component of $\sigma_{x x}$ may have non-zero value, because edge effects have not been taken into account. The same explanation can be given for the $\sigma_{y y}$ stress component. Even if the average value of $\sigma_{y y}$ can be considered as negligible, its local value is non-zero as specimen with large transversal dimensions under uniaxial compressive loadings involves local transversal stresses, except for null effective Poisson ratio materials. Because the present experiments do not allow obtaining these local variables, the assumption of zero value for $\sigma_{x x}$ and $\sigma_{y y}$ has been made.

The logarithmic strain tensor obtained using the D.I.C. technique, which is presented in equation (2), involves only two dimensions. Assuming that we were dealing here with macroscopically homogeneous material, $\varepsilon x y$ and $\varepsilon x z$ could be taken to be equal to 0 under both loading conditions.

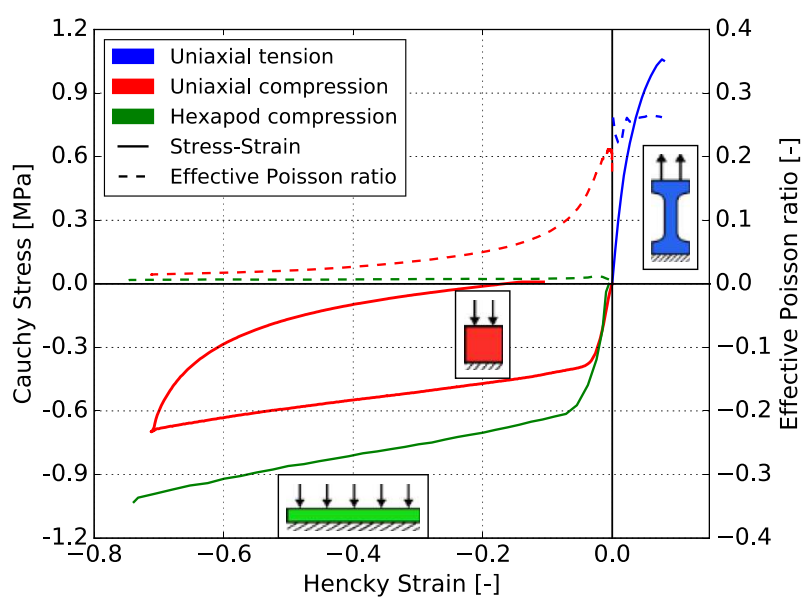

Fig. 4. Uniaxially loaded EPP foam at a strain rate of $0.0125 \mathrm{~s}^{-1}$.

$\left[\varepsilon_{i j}\right]=\left[\begin{array}{ccc}\varepsilon_{x x} & 0 & 0 \\ 0 & \varepsilon_{y y} & \varepsilon_{y z} \\ 0 & \varepsilon_{y z} & \varepsilon_{z z}\end{array}\right]$

A second camera was placed perpendicularly to the first one in order to measure the transversal strain. The transversal strain $\varepsilon x x$ did not exceed $0.6 \%$ in any of the simple shear tests, and was therefore not significant. For the sake of simplicity, the $\varepsilon_{x x}$ strain component was therefore set at 0 in the simple shear and uniaxial compression tests. Section 3.1 gives further information about the effective Poisson ratio in the case of tensile tests. Other components of the strain tensor (see equation (2)) were calculated from the results obtained using the D.I.C. technique.

\subsubsection{Choice of the post-processing framework}

Multiaxial experiments are complex tests from which it can be difficult to extract useful data. The classical stress-strain curve in Fig. 4 is not appropriate for comparing various loads or determining the responses of the material to multiaxial loads. One way of simplifying the analysis consists in studying the elementary mechanical behavior by separating the volume change responses from the shape change responses, in line with Combaz et al. [18,19]. These two contributions have been classically defined as the spherical and deviatoric parts of the stress and strain tensors, as long as suitable tensors are chosen. Due to the large transformations occurring in EPP foam, Criscione et al. [17] have recommended using the Cauchy stress tensor associated with the Hencky logarithmic strain tensor.

The volume and shape changes can be separated by performing a spherical/deviatoric decomposition: $\widetilde{\sigma}=\widetilde{\sigma}^{s}+\widetilde{\sigma}^{d}$ and $\tilde{\varepsilon}=\tilde{\varepsilon}^{s}+\widetilde{\varepsilon}^{d}$, where the superscripts.$^{s}$ and.$^{d}$ denote the spherical and deviatoric contributions, respectively. The invariants of the tensor $\tilde{a}$ used in the present study were:

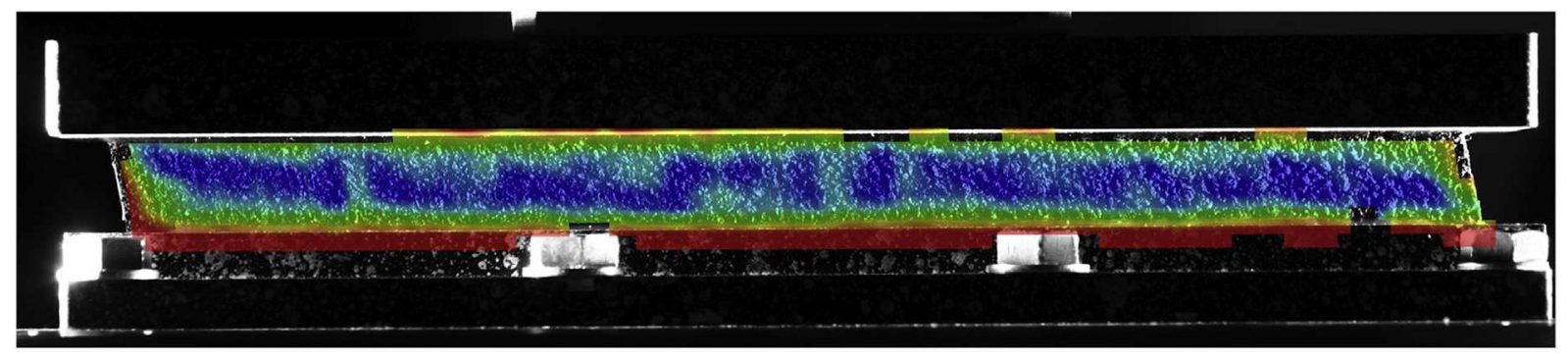

Fig. 3. Normalized strain field $\varepsilon_{y z}$ obtained by D.I.C. analysis. The region of interest was chosen with a view to ruling out the occurrence of any boundary effects. 


$$
\left\{\begin{array}{c}
I_{\widetilde{a}}=\operatorname{tr}(\widetilde{a}) \\
I I_{\widetilde{a}}=\frac{1}{2}\left(\left(I_{\widetilde{a}}\right)^{2}-\operatorname{tr}\left(\widetilde{a}^{2}\right)\right) \\
I I I_{\widetilde{a}}=\operatorname{det}(\widetilde{a})
\end{array}\right.
$$

The volume change contribution was assessed from the pressure $p$ versus the relative volume $V$. Relations between these two variables can be expressed as follows, taking the first invariant of the stress tensor, $I_{\tilde{\sigma}}$, or the strain tensor, $I_{\tilde{\varepsilon}}$ :

$p=-\frac{I_{\widetilde{\sigma}}}{3} \quad ; \quad V=\frac{v}{v_{0}}=\exp \left(I_{\tilde{\varepsilon}}\right)$

where $v$ and $v 0$ are the current and initial volume, respectively.

The shape change contribution, which is complementary to the volume change contribution, can be described by:

- the intensities of the deviatoric tensors, the deviatoric stress intensity $Q \sigma$ and the deviatoric strain intensity $Q \varepsilon$, based on the second invariant of their deviatoric tensors, $I I_{\widetilde{\sigma}^{d}}$ and $I I_{\tilde{\varepsilon}^{d}}$, respectively.

$Q_{\sigma}=\sqrt{\widetilde{\sigma}^{d}: \widetilde{\sigma}^{d}}=\sqrt{-2 I I_{\widetilde{\sigma}^{d}}} \quad ; \quad Q \varepsilon=\sqrt{\widetilde{\varepsilon}^{d}: \widetilde{\varepsilon}^{d}}=\sqrt{-2 I I_{\tilde{\varepsilon}^{d}}}$

- the directions in the deviatoric planes, which are given by the Lode angles $\varphi \sigma$ and $\varphi \varepsilon$ defined in terms of the third and second invariants of the stress and strain deviatoric tensors, respectively.

$\varphi_{\sigma}=\frac{1}{3} \arccos \left(3 \sqrt{6} \frac{I I I_{\tilde{\sigma}^{d}}}{\left(-2 I I_{\widetilde{\sigma}^{d}}\right)^{\frac{3}{2}}}\right) \quad ; \quad \varphi_{\varepsilon}=\frac{1}{3} \arccos \left(3 \sqrt{6} \frac{I I I_{\tilde{\varepsilon}^{d}}}{\left(-2 I I_{\tilde{\varepsilon}^{d}}\right)^{\frac{3}{2}}}\right)$

One of the advantages of using mathematical tensor invariants is that this makes it possible to compare mechanically relevant variables which do not depend on the coordinate system used. In the case of the experiments presented in section 2.2.1, it was therefore possible to calculate the volume change and shape change variables from the experimental data as follows:

$$
\left\{\begin{array}{c}
p=-\frac{\sigma_{z z}}{3} \\
Q_{\sigma}=\sqrt{\frac{2}{3} \sigma_{z z}^{2}+2 \sigma_{y z}^{2}} \\
\varphi=\frac{1}{3} \arccos \left(\frac{\left(\frac{2}{3} \sqrt{\frac{2}{3}} \sigma_{z z}^{3}+\sqrt{6} \sigma_{z z} \sigma_{y z}^{2}\right)}{\left(\frac{2}{3} \sigma_{z z}^{2}+2 \sigma_{y z}^{2}\right)^{\frac{3}{2}}}\right)
\end{array}\right.
$$

$\left\{\begin{array}{c}V=\exp \left(\varepsilon_{x x}+\varepsilon_{y y}+\varepsilon_{z z}\right) \\ Q_{\varepsilon}=\sqrt{\frac{2}{3}\left[\left(\varepsilon_{x x}-\varepsilon_{y y}\right)^{2}+\left(\varepsilon_{y y}-\varepsilon_{z z}\right)^{2}+\left(\varepsilon_{z z}-\varepsilon_{x x}\right)^{2}\right]+2 \varepsilon_{y z}^{2}} \\ \varphi_{\varepsilon}=\frac{1}{3} \arccos \left(\frac{\frac{\sqrt{6}}{9}\left[\left(\varepsilon_{x x}-\varepsilon_{y y}\right)^{3}+\left(\varepsilon_{y y}-\varepsilon_{z z}\right)^{3}+\left(\varepsilon_{z z}-\varepsilon_{x x}\right)^{3}\right]+\sqrt{6} \varepsilon_{y z}^{2}\left(-2 \varepsilon_{x x}+\varepsilon_{y y}+\varepsilon_{z z}\right)}{\left(\frac{2}{3}\left[\left(\varepsilon_{x x}-\varepsilon_{y y}\right)^{2}+\left(\varepsilon_{y y}-\varepsilon_{z z}\right)^{2}+\left(\varepsilon_{z z}-\varepsilon_{x x}\right)^{2}\right]+2 \varepsilon_{y z}^{2}\right)^{\frac{3}{2}}}\right)\end{array}\right.$

It is also worth defining two other geometric variables to make easier the understanding of strain and stress paths. The kinematic angle $\theta \varepsilon$ can be defined as the elevation of the volume related to the deviatoric strain plane. With the same manner, the stress angle $\theta_{\sigma}$ can be defined as the elevation of the pressure related to the deviatoric stress plane, and corresponds to the triaxiality value. These two variables give meaningful information on the volume change compared to the shape change and will be defined in the following sections.

\section{Basic experiments}

\subsection{Effective Poisson ratio}

Mechanical characterization of cellular materials is usually conducted by performing uniaxial compression tests. To obtain failure data, uniaxial tensile tests can also be performed. The stress vs. strain graph obtained with the present EPP material is given by the full colored curves in Fig. 4. The compression response of the EPP material shows the presence of a large reversible contribution and a large hysteresis loop when loading - unloading was performed.

Another parameter often used to characterize cellular materials' behavior is the transversal changes occurring during uniaxial loading, which have been assimilated to an effective Poisson ratio. This parameter is known to be non-constant and often amounts to almost zero under uniaxial compression loading conditions $[27,28]$, which can be explained by micro-structural features of the material with the works of Gibson and Ashby [29] or more recently with the numerical works of Mihai and Goriely [30]. Indeed, under compressive loadings, the foam cells collapse, by a buckling process of the cell walls, in the free space of the cell and this involves a low effective Poisson ratio. Under tensile loading, the process is different. The foam cells are stretched and this involves a contraction of the cell in the transversal direction and a nonnull Poisson ratio. Under simple shear loading, foam cells are subjected to both compression and tension. Equilibrium of the foam under simple shear loading involves a competition between tension and compression processes in terms of transversal strain.

Based on the images recorded during the tests, transversal strains have been measured in order to obtain the effective Poisson ratio. As was to be expected and explained above, in line with findings made by Widdle et al. [28], the effective Poisson ratio of the present EPP material varies during uniaxial loading (see the dotted colored curves in Fig. 4). It amounts to approximately 0.27 during uniaxial tension and ranges from 0.27 to 0.03 during uniaxial compression. When a specimen for hexapod device is used, the effective Poisson ratio is very different from a specimen for classical electromechanical device (see dotted green curve in Fig. 4). Indeed, the effective Poisson ratio is almost equal to 0.02 since the beginning of the compressive test. This is due to the size of the specimen that limits transversal displacements and also involves a stress increase (see full green curve in Fig. 4).

As explained in section 2.2.2, the assumption that $\varepsilon x x=0$ in the case of uniaxial compression and simple shear was adopted here for the sake of simplicity. However, this assumption cannot be made in the case of uniaxial tensile tests. The strain components $\varepsilon x x$ and $\varepsilon y y$ were therefore calculated using an effective Poisson ratio of 0.27 to be in line with the experimental values. All the strain invariants in equation (8) were therefore calculated on the basis of these assumptions.

\subsection{Comparisons between basic experiments}

The results of uniaxial compression, simple shear and uniaxial tension tests were expressed in the post-processing framework presented in section 2.2.3 in terms of the volume change and shape change contributions. Uniaxial compression and simple shear tests were performed using the hexapod device and the specimen described in section 2.1.2. Uniaxial tension tests were performed using a conventional electromechanical device and a specimen corresponding to French standard NF EN ISO 1798 [21]. All these experiments were performed with a strain rate $\dot{\varepsilon}=0.0125 \mathrm{~s}^{-1}$.

The repeatability of all the tests was checked. Three specimens at least were tested in each case. As shown in Fig. 5, the results obtained in the experiments of each kind showed good repeatability. The slight variations observed were due to the density of the specimens, which affects the mechanical behavior of foam [29] but did not invalidate the results of the analysis. The onset of rupture of the specimen is indicated by pink stars on all the graphs presented in this paper.

The contribution of volume changes is presented in Fig. $5 \mathrm{a}$ in all the basic tests. Uniaxial compression resulted in a decrease in the volume of the specimen and an increase in the pressure (red curves in Fig. 5a). A pressure threshold can be clearly observed at a pressure $p=0.2 \mathrm{MPa}$ and a volume ratio $V=0.98$. This threshold matches the behavioral change observed on the microscopic scale between the elastic porosity 


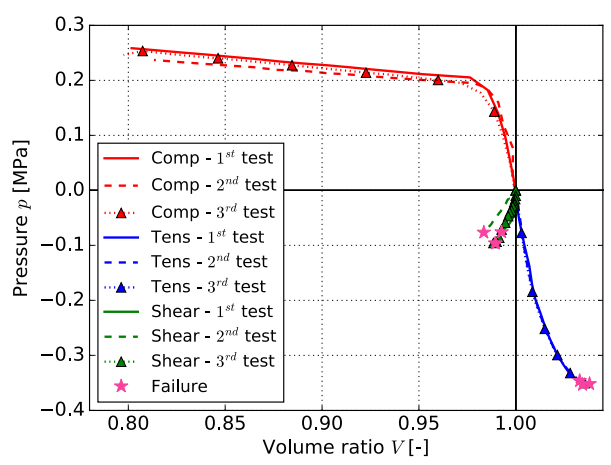

(a) Volume change contribution.

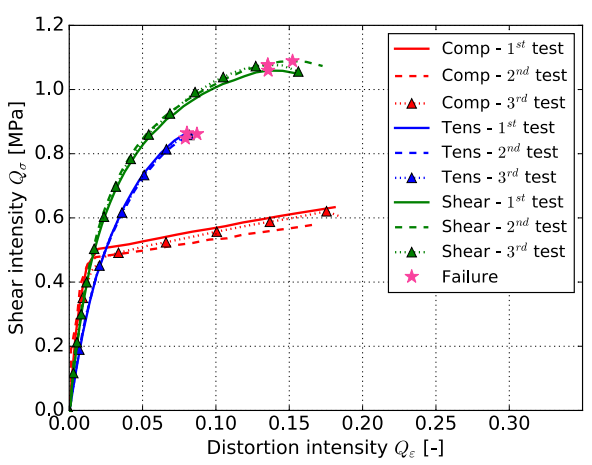

(b) Shape change contribution.

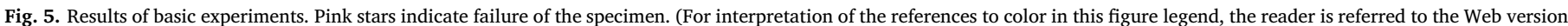
of this article.)

stage and the stress plateau stage [29]. Uniaxial tension resulted in an increase in the volume and a decrease in the pressure until failure occurred at a pressure $p=-0.32 \mathrm{MPa}$ (see pink stars on blue curves in Fig. 5a). Simple shear resulted in a drop of $0.08 \mathrm{MPa}$ in the pressure, as shown by the green curves in Fig. 5a. This drop was due to the nature of the simple shear load, which was not a pure shear load. A slight decrease in the volume was also observed in the simple shear test, due to issues concerning the rigidity of the device. The effects of the rigidity of the device were corrected and reduced in all the shear tests by adjusting the movements of the hexapod platform. An arbitrary criterion defining valid tests was set at $2 \%$ of the maximum volume change.

The contribution of the shape changes is presented in Fig. 5b. In the case of uniaxial compression, a shear threshold was observed at a shear intensity $Q \sigma=0.5 \mathrm{MPa}$ and a distortion intensity $Q \varepsilon=0.02$, which matches the pressure threshold observed in the case of the volume change behavior. The shape change behavior corresponding to a simple shear load was nearly the same as under uniaxial tension, but at a higher shear intensity. This difference can originate in the mechanical behavior of polymers that may be different in tension and in compression. But it mainly originates in specimen geometries that involve non-null local transversal stress $\sigma_{y y}$, especially near the grip, for hexapod device specimens and higher stiffness. Failure occurred at higher stress and strain intensities in the simple shear tests, $Q \sigma=1.1 \mathrm{MPa}$ as compared to $0.8 \mathrm{MPa}$ and $Q \varepsilon=0.15$ as compared to 0.07 under uniaxial tension, respectively.

\section{Multiaxial experiments}

\subsection{Experimental procedure}

We then focused on the influence of the volume change behavior on the shape change behavior, and vice-versa. Several multiaxial tests were performed in which radial loading paths were applied. In each of these tests, the kinematics imposed on the specimen using the hexapod followed a radial path, which can be defined as the kinematic angle $\theta \varepsilon$ between the ordinate axis $Q \varepsilon$ and the radial path (see equation (9)).

$\theta_{\varepsilon}=\arctan \left(\frac{\ln V}{Q_{\varepsilon}}\right)=\arctan \left(\frac{I_{\varepsilon}}{Q_{\varepsilon}}\right)$

For instance, the kinematic angle was equal to $0^{\circ}$ in the simple shear tests and $-90^{\circ}$ or $+90^{\circ}$ in the triaxial compression and tension tests, respectively. Using equation (8), the volume ratio $I \varepsilon$ and the distortion intensity $Q \varepsilon$ can be expressed as a function of the stretch value $\lambda$ and the effective Poisson ratio $\alpha$, giving $\theta \varepsilon$ under uniaxial loading conditions (see equation (10)). $\theta_{\varepsilon}=\arctan \left(\frac{(1-2 \alpha) \ln \lambda}{(1+\alpha)|\ln \lambda| \sqrt{\frac{2}{3}}}\right)$

Two new radial paths in which simultaneous uniaxial compression and simple shear are combined can be arbitrary defined. The combined tests \#1 and \#2 followed radial loading paths. Their specific kinematic angles are defined between uniaxial compression and simple shear, and are equal to $-42^{\circ}$ and $-31^{\circ}$, respectively. The characteristics of each test are summarized in Table 1.

\subsection{Results}

\subsubsection{Kinematic behavior observed}

The radial paths obtained in the D.I.C. analysis were presented in a diagram giving the distortion intensity vs. one of the volume variables, as shown in Fig. 6.

When the results were presented in a $Q \varepsilon-V$ diagram (see Fig. 6a), the curves obtained were not completely straight because of the choice of variable $V=\exp (I \varepsilon)$. However, when the results of the tests were presented as in the $Q \varepsilon-I \varepsilon$ diagram in Fig. $6 \mathrm{~b}$, they exactly matched the radial paths applied although the Combined 1 test did not give a completely straight line. This was probably due to the large transformations imposed on the hexapod device, which had some difficulty in following the imposed loading path.

Failure of the specimens (pink stars on the graphs) occurred in all the tests except the uniaxial compression test. In tests with a low kinematic angle, failure in terms of the distortion intensity was delayed. These diagrams are useful means of defining failure limit curves because the failure process evolves very regularly with the kinematic angle. Linear and quadratic polynomial functions can therefore be used to fit the failure limit curve in both cases. Results of fitting procedure are presented in Table 2. It is worth noting that the use of a linear function gives an accurate approximation with a coefficient of determination $R^{2}$ greater than 0.99 .

Table 1

Controlled radial path experiments.

\begin{tabular}{ll}
\hline Loading & Kinematic angle $\theta_{\varepsilon}$ \\
\hline Uniaxial compression $(\alpha=0)$ & $-51^{\circ}$ \\
Combined 1 & $-42^{\circ}$ \\
Combined 2 & $-31^{\circ}$ \\
Simple shear & $0^{\circ}$ \\
Uniaxial tension $(\alpha=0.27)$ & $24^{\circ}$ \\
\hline
\end{tabular}




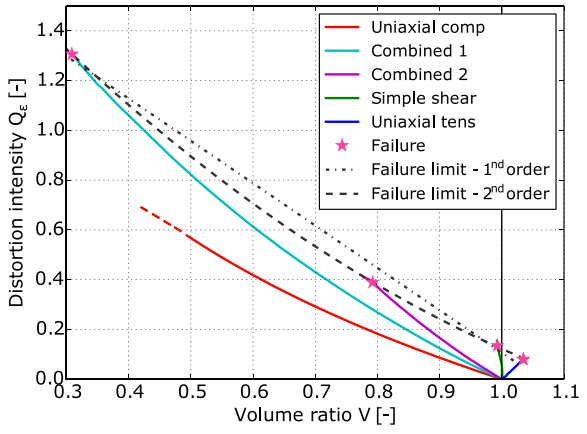

(a) Kinematics using the $V$ variable

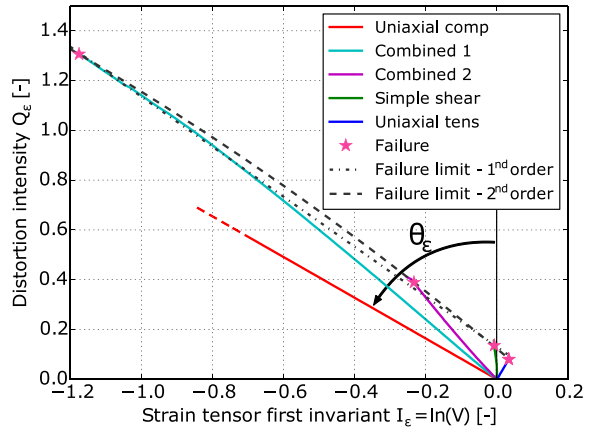

(b) Kinematics using the $I_{\varepsilon}$ variable

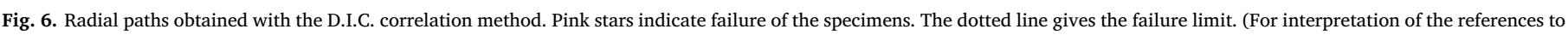
color in this figure legend, the reader is referred to the Web version of this article.)

Table 2

Failure limit functions.

\begin{tabular}{lll}
\hline $\begin{array}{l}\text { Polynomial } \\
\text { functions }\end{array}$ & Equation & $\begin{array}{l}\text { Coefficient of } \\
\text { determination } \boldsymbol{R}^{2}\end{array}$ \\
\hline Linear & $Q_{\varepsilon}=-1.702 V+1.808$ & 0.9929 \\
& $Q_{\varepsilon}=-1.004 I_{\varepsilon}+0.131$ & 0.9991 \\
Quadratic & $Q_{\varepsilon}=0.870 V^{2}-2.851 V+2.103$ & 0.9999 \\
& $Q_{\varepsilon}=-0.146 I_{\varepsilon}^{2}-1.178 I_{\varepsilon}+0.123$ & 1.0000 \\
\hline
\end{tabular}

\subsubsection{Volume change and shape change behavior}

Three multiaxial experiments of each kind were performed to check the repeatability. Results are presented in terms of volume and shape changes in Fig. 7a and b, respectively. The scattering of the data was moderate and the main differences between results were due to differences between the density of the specimens.

Since it is proposed in the future to model the mechanical behavior of foams by separating the volume changes from the shape changes, it is worthwhile having some parameters which describe each of these contributions. In the above two figures, the curves look alike, and most of them are composed of two different slopes, corresponding to a threshold and a failure limit. To facilitate the observation and analysis of these curves, several parameters have been defined in Table 3.

In terms of the volume changes, the first slope $K 1$ was nearly the same in all the tests in which uniaxial loading was applied. This slope differed in the case of the simple shear tests, however, because of issues concerning the rigidity of the experimental device, as mentioned in section 3.2. The kinematic angle was found to affect the pressure threshold $P \sigma 0$ and the second slope $K 2$, and hence the volume change behavior. When $\theta_{\varepsilon}$ increased, the pressure threshold $P \sigma 0$ decreased and
Table 3

Definition of parameters.

\begin{tabular}{lll}
\hline Description & Parameters Volume change & Parameters Shape change \\
\hline First slope & $K_{1}$ & $\mu_{1}$ \\
Threshold & $P_{\sigma}^{0}$ & $Q_{\sigma}^{0}$ \\
Second slope & $K_{2}$ & $\mu_{2}$ \\
Failure & $V^{f}$ and $P_{\sigma}^{f}$ & $Q_{\varepsilon}^{f}$ and $Q_{\sigma}^{f}$ \\
\end{tabular}

Table 4

Effects of the kinematic angle on the volume change behavior.

\begin{tabular}{llllll}
\hline Loading $\left(\theta_{\varepsilon}\right)$ & $K 1[\mathrm{MPa}]$ & $\boldsymbol{P}_{\sigma}^{0}[\mathrm{MPa}]$ & $\boldsymbol{K} 2[\mathrm{MPa}]$ & $\boldsymbol{V}^{\boldsymbol{f}}[-]$ & $\boldsymbol{P}_{\sigma}^{f}[\mathrm{MPa}]$ \\
\hline Uniaxial compression & -11.5 & 0.21 & -0.28 & - & - \\
$\quad\left(-51^{\circ}\right)$ & & & & & \\
Combined 1 $\left(-42^{\circ}\right)$ & -11.7 & 0.15 & +0.12 & 0.28 & 0.26 \\
Combined 2 $\left(-21^{\circ}\right)$ & -11.8 & 0.13 & +0.59 & 0.77 & 0.03 \\
Simple shear $\left(0^{\circ}\right)$ & - & - & - & 0.99 & -0.08 \\
Uniaxial tension $\left(24^{\circ}\right)$ & - & - & - & 1.04 & -0.35 \\
\hline
\end{tabular}

the second slope $K 2$ also decreased relatively. Except for the uniaxial compression tests, in which no failure occurred, the pressure at failure $P \sigma f$ decreased when $\theta \varepsilon$ increased. All the results showing the effects of the kinematic angle on the volume change behavior are presented in Table 4.

In terms of the shape changes, the first slope $\mu 1$ was practically nearly the same in all the basic and multiaxial tests, except for the uniaxial tensile test. This difference in stiffness can be explained, firstly, by the different mechanical behaviors in tension and compression of

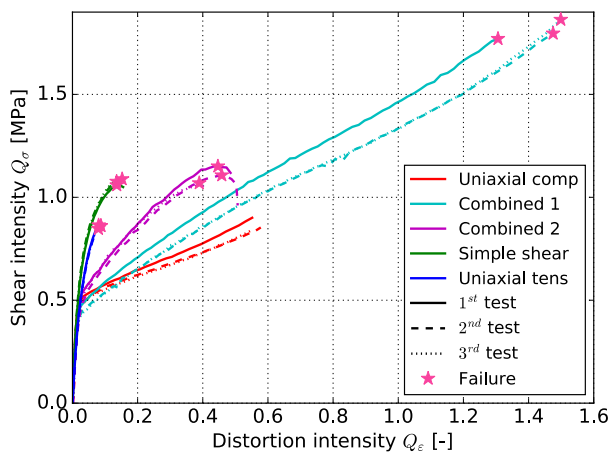

(b) Shape change contribution

\section{(a) Volume change contribution}

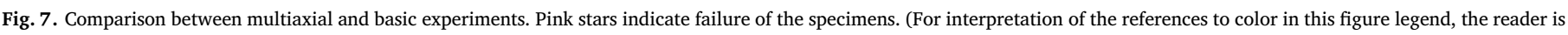
referred to the Web version of this article.) 
some polymers and, secondly, by the different specimen geometry used for these two loadings, as mentioned in section 3.2.

As regards the threshold and the second slope, it is worth noting that in all the tests including a uniaxial compression loading contribution, i.e., the uniaxial compression test, the combined \#1 and the combined \#2 tests, the threshold was approximately the same at about $Q_{\sigma}^{0}=0.5 \mathrm{MPa}$. In these three tests, the second slope subsequently increased when the kinematic angle increased. In a future objective of developing a new numerical model to describe EPP mechanical behavior or more generally foam mechanical behavior, a first process step is to define mathematical functions that can describe both volume change and shape change contributions. If the mathematical functions are the same whatever the kind of loadings (tension, compression, shear), the second process step which consisting in defining a hyperelastic potential with separation of spherical and deviatoric contributions will become easier. If we take this point further by increasing the kinematic angle, assuming the existence of a shear threshold of $0.5 \mathrm{MPa}$ in all the tests, including the simple shear and tensile tests, the second slope will therefore increase reaching a maximum in the simple shear tests. This assumption might help to explain the shape of these two curves.

Lastly, decreasing the kinematic angle delayed the failure in terms of the distortion intensity and the shear intensity. All the results showing the effects of the kinematic angle on the shape change behavior are presented in Table 5.

\subsubsection{Stress response of the material}

Just as the kinematic angle $\theta \varepsilon$ defines the kinematics imposed on the specimen, the stress angle $\theta \sigma$ defined in equation (11) reflects the evolution of the loading in terms of the shear rate and the pressure, and corresponds almost to the triaxiality value.

$\theta_{\sigma}=\arctan \left(\frac{p}{Q_{\sigma}}\right)$

For instance, in the case of a simple shear load, $\theta \sigma=0^{\circ}$ under uniaxial loading, the stress angle can be calculated as in equation (12).

$\theta_{\sigma}=\arctan \left(\frac{-\frac{\sigma}{3}}{|\sigma| \sqrt{\frac{2}{3}}}\right) \simeq \pm 22^{\circ}$

It is worth presenting the evolution of the specimen under different loads in a 'Shear intensity $Q \sigma$ - Pressure $p$ ' diagram such as that shown in Fig. 8.

It is worth noting that the uniaxial tension and compression loads gave straight paths. In the simple shear tests, the stress angle was not exactly null because the load applied was not a pure shear load (see section 3.2). In the combined tests \#1 and \#2, the initial slopes were equal to $18^{\circ}$ and $13^{\circ}$, respectively. A sharp change then occurred at $Q_{\sigma}^{\# 1}$ $=0.49 \mathrm{MPa}$ and $Q_{\sigma}^{\# 2}=0.55 \mathrm{MPa}$ at the same shear and pressure thresholds. From then on, the loading path made a change of direction, resembling that obtained with uniaxial tension or simple shear loads with stress angles of $\theta_{\sigma}^{\# 1}=-4^{\circ}$ and $\theta_{\sigma}^{\# 2}=-10^{\circ}$. Only the combined test \#2 gradually resulted in another change of direction, resembling that observed under uniaxial compression loading just before failure of the specimen occurred. One important point worth noting is that with

Table 5

Effects of the kinematic angle on the shape change behavior.

\begin{tabular}{llllll}
\hline Loading $\left(\theta_{\varepsilon}\right)$ & $\mu 1[\mathrm{MPa}]$ & $Q_{\sigma}^{0}[\mathrm{MPa}]$ & $\mu 2[\mathrm{MPa}]$ & $Q_{\varepsilon}^{f}[-]$ & $Q_{\sigma}^{f}[\mathrm{MPa}]$ \\
\hline $\begin{array}{l}\text { Uniaxial compression } \\
\quad 34.1\end{array}$ & 0.51 & 0.66 & - & - \\
$\quad\left(-51^{\circ}\right)$ & & & & & \\
Combined 1 $\left(-42^{\circ}\right)$ & 31.0 & 0.46 & 1.17 & 1.43 & 1.81 \\
Combined 2 $\left(-21^{\circ}\right)$ & 30.0 & 0.55 & 1.83 & 0.43 & 1.11 \\
Simple shear $\left(0^{\circ}\right)$ & 32.7 & $(0.47)$ & $(11.15)$ & 0.14 & 1.08 \\
Uniaxial tension $\left(24^{\circ}\right)$ & 22.3 & $(0.55)$ & $(7.33)$ & 0.08 & 0.86 \\
\hline
\end{tabular}

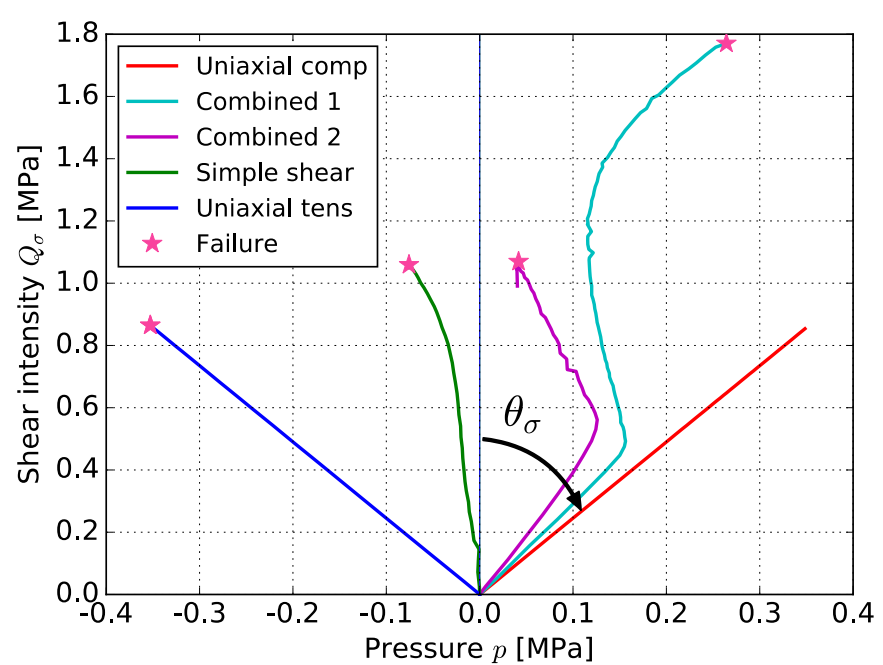

Fig. 8. Loading paths obtained with the $3 \mathrm{D}$ sensor. Pink stars stand for failure of the specimen. (For interpretation of the references to color in this figure legend, the reader is referred to the Web version of this article.)

straight imposed kinematic paths (see Fig. 6), the loading paths obtained were not all straight. The great differences in behavior observed between the uniaxial compression and simple shear tests (see section 3) resulted in changes in the loading paths when both types of loading were combined. This key point will be confirmed in the following subsection entitled 'Strain and stress Lode angles'. Defining a failure criterion seems to be more difficult with the stress variables $p$ and $Q \sigma$ in Fig. 8 than with the strain variables $V$ or $I \varepsilon$ and $Q \varepsilon$ in Fig. 6.

\subsubsection{Stress and strain Lode angles}

The loading paths in the stress or strain deviatoric planes can be approached using the strain and stress Lode angles as defined in section 2.2.3 and shown in Fig. 9.

All the changes in the strain Lode angles shown in Fig. 9a corresponded to straight paths. Under uniaxial tension and compression, the Lode angles $\varphi \varepsilon=0^{\circ}$ and $\varphi \varepsilon=60^{\circ}$, respectively, which is in agreement with the theoretical values. In the case of simple shear loads, the Lode angle calculated using the Digital Image Correlation method amounted to the expected value of $30^{\circ}$. In the combined tests $\# 1$ and $\# 2$, the strain Lode angles obtained were approximately equal to $45^{\circ}$ and $50^{\circ}$, respectively.

For the same reasons as those mentioned in section 4.2.3, none of the stress Lode angles gave completely straight paths, except for those obtained with uniaxial tensile and compression loads. The stress Lode angle obtained in the simple shear test gradually departed from the shear angle, mainly because pure shear loads were not applied. In the combined tests, since the simple shear and uniaxial compressive contributions were very different, the stress Lode angle suddenly changed when the shear threshold was reached. This change of behavior was confirmed by the elevation related to the deviatoric stress plane (Fig. 8) and the Lode angle on the deviatoric stress plane (Fig. 9b). Failure, which may depend on either the stress or strain Lode angles, occurred earlier in the case of low Lode angles.

\section{Conclusions}

In this study, original multiaxial experiments were performed using a novel hexapod device, with which uniaxial compression and simple shear loads can be applied simultaneously to a polymeric foam to test its mechanical behavior with radial loading paths. The Cauchy stress tensor was obtained using a 3-D sensor and the logarithmic strain tensor using the Digital Image Correlation method. The evolution of the effective Poisson ratio was determined and the assumptions adopted in 


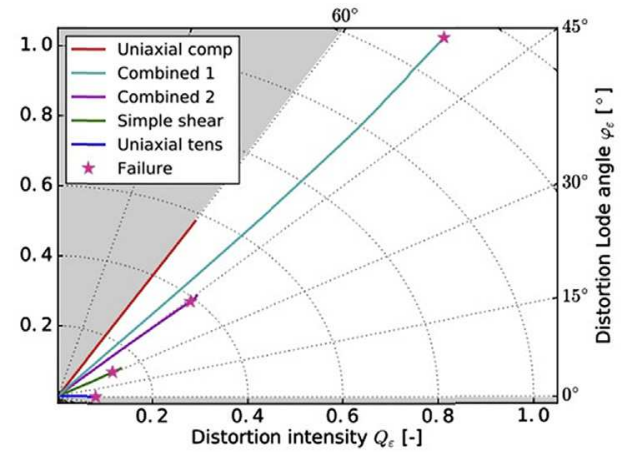

(a) Strain contribution

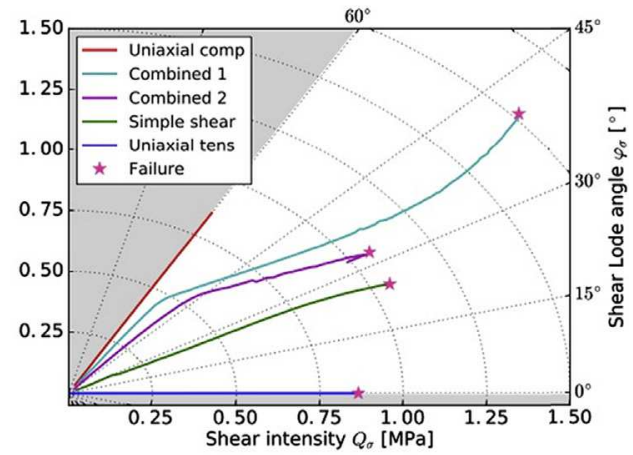

(b) Stress contribution

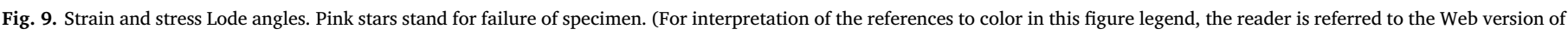
this article.)

order to obtain inaccessible strain components are discussed.

The post-processing framework based on tensor invariants used by Combaz et al. [18,19] was adopted for comparing the results of basic and multiaxial experiments.

The results obtained in this study can be summarized as follows:

1. The mechanical behavior depends on the kinematic angle, and hence on the contribution of the simple shear to combined loads. The results of a detailed study show the effects of this parameter on the various steps in the mechanical response of the material, decomposed into the volume and shape change contributions. In short, - the elasticity of microscopic cell walls, as defined by Gibson and Ashby [29], is not subject to any variations depending on the kinematic angle;

- the pressure threshold decreases with the kinematic angle, whereas the shear threshold does not show any changes;

- the pressure resistance observed during the stress plateau evolves in the opposite way from the shear resistance.

2. Failure occurred later in the case of combined loads involving relative small simple shear contributions. The failure limit can be easily determined in terms of the strain path using a distortion intensity-to-volume ratio diagram.

3. The radial strain paths imposed did not systematically result in straight stress paths. Since the mechanical behavior of the material differed considerably between uniaxial compression and simple shear tests, the stress paths observed in the combined tests were not radial in terms of either the pressure-to-shear intensity ratio or the stress Lode angle.

In conclusion, the results obtained in this study increase the database available on this widely used foam, which will also be extremely useful in our future project, in which it is proposed to model these experiments. In particular, the possibility to get the loading path in the stress space related to the strain space brings useful information. Since the novel hexapod device can be used to perform multiaxial tests, it is also intended to perform tests of this kind with various loading paths in order to determine how they affect the mechanical behavior of the material of interest.

\section{Acknowledgments}

The French Fonds Unique Interministériel (FUI number F 1410009 M) is gratefully acknowledged for funding the New Generation of Airliner Seats (Genosia) project.

\section{References}

[1] N.J. Mills, C. Fitzgerald, A. Gilchrist, R. Verdejo, Polymer foams for personal protection: cushions, shoes and helmets, Compos. Sci. Technol. 63 (16) (2003) 2389-2400.

[2] F.A.O. Fernandes, R.J. Alves de Sousa, Motorcycle helmets-a state of the art review, Accid. Anal. Prev. 56 (2013) 1-21.

[3] L.M. Yang, V.P.W. Shim, A visco-hyperelastic constitutive description of elastomeric foam, Int. J. Impact Eng. 30 (8) (2004) 1099-1110 Eighth International Symposium on Plasticity and Impact Mechanics (IMPLAST 2003).

[4] J. Zhang, N. Kikuchi, V. Li, A. Yee, G. Nusholtz, Constitutive modeling of polymeric foam material subjected to dynamic crash loading, Int. J. Impact Eng. 21 (5) (1998) 369-386.

[5] P. Viot, F. Beani, J.-L. Lataillade, Polymeric foam behavior under dynamic compressive loading, J. Mater. Sci. 40 (2005) 5829-5837.

[6] S. Ouellet, D. Cronin, M. Worswick, Compressive response of polymeric foams under quasi- static, medium and high strain rate conditions, Polym. Test. 25 (6) (2006) 731-743.

[7] R. Bouix, P. Viot, J.-L. Lataillade, Polypropylene foam under dynamic loadings : strain rate, density and microstructure effects, Int. J. Impact Eng. 36 (2) (2009) 329-342.

[8] R.W. Ogden, G. Saccomandi, I. Sgura, Fitting hyperelastic models to experimental data, Comput. Mech. 34 (6) (Nov 2004) 484-502.

[9] M. Mooney, A theory of large elastic deformation, J. Appl. Phys. 11 (1940) 582.

[10] R.S. Rivlin, Large elastic deformations of isotropic materials iv. further developments of thegeneral theory, Phil. Trans. Roy. Soc. 241 (835) (1948) 379-397.

[11] Y. Masso Moreu, N.J. Mills, Rapid hydrostatic compression of low-density polymeric foams, Polym. Test. 23 (3) (2004) 313-322.

[12] P. Viot, Hydrostatic compression on polypropylene foam, Int. J. Impact Eng. 36 (7) (2009) 975-989.

[13] U.E. Ozturk, G. Anlas, Hydrostatic compression of anisotropic low density polymeric foamsunder multiple loadings and unloadings, Polym. Test. 30 (2011) $737-742$.

[14] A. Krundaeva, D. De Bruyne, F. Gagliardi, W. Van Paepegem, Dynamic compressive strength and crushing properties of expanded polystyrene foam for different strain rates and different temperatures, Polym. Test. 55 (2016) 61-68.

[15] M. Kintscher, L. Kärger, A. Wetzel, D. Hartung, Stiffness and failure behaviour of folded sandwich cores under combined transverse shear and compression, Compos. Appl. Sci. Manuf. 38 (5) (2007) 1288-1295.

[16] Y. Mosleh, J. Vander Sloten, B. Depreitere, J. Ivens, Novel composite foam concept for head protection in oblique impacts, Adv. Eng. Mater. (2017) 1700059.

[17] J.C. Criscione, J.D. Humphrey, A.S. Douglas, W.C. Hunter, An invariant basis for natural strain which yields orthogonal stress response terms in isotropic hyperelasticity, J. Mech. Phys. Solid. 48 (2000) 2445-2465.

[18] E. Combaz, C. Bacciarini, R. Charvet, W. Dufour, F. Dauphin, A. Mortensen, Yield surface of polyurethane and aluminium replicated foam, Acta Mater. 58 (15) (2010) 5168-5183.

[19] E. Combaz, C. Bacciarini, R. Charvet, W. Dufour, A. Mortensen, Multiaxial yield behaviour of al replicated foam, J. Mech. Phys. Solid. 59 (9) (2011) 1777-1793.

[20] L. Maheo, S. Guérard, G. Rio, A. Donnard, P. Viot, Multiaxial behavior of foams experiments and modeling, 11th Int DYMAT Conference. EPJ Web Of Conferences 94 (2015), p. 04035.

[21] AFNOR. NF EN ISO 1798, Matériaux polymères alvéolaires souples - Détermination de la résistance à la traction et de l'allongement à la rupture, (April 2008).

[22] A. Wada, T. Kawasaki, Y. Minoda, A. Kataoka, S. Tashiro, H. Fukuda, A method to measure shearing modulus of the foamed core for sandwich plates, Compos. Struct. 60 (2003) 385-390.

[23] E.W. Andrews, G. Gioux, P. Onck, L.J. Gibson, Size effects in ductile cellular solids. Part II : experimental results, Int. J. Mech. Sci. 43 (2001) 701-713.

[24] C. G'Sell, S. Boni, S. Shrivastava, Application of the plane simple shear test for determination of the plastic behaviour of solid polymers at large strains, J. Mater. Sci. 18 (1983) 903-918.

[25] S. Bouvier, H. Haddadi, P. Levée, C. Teodosiu, Simple shear tests : experimental techniques and characterization of the plastic anisotropy of rolled sheets at large strains, J. Mater. Process. Technol. 172 (2006) 96-103. 
[26] A. Mostafa, K. Shankar, E.V. Morozov, Effect of shear keys diameter on the shear perfor-mance of composite sandwich panel with PVC and PU foam core : FE study, Compos. Struct. 102 (2013) 90-100.

[27] J.B. Choi, R.S. Lakes, Nonlinear properties of polymer cellular materials with a negative Poisson's ratio, J. Mater. Sci. 27 (1992) 4678-4684.

[28] R.D. Widdle, A.K. Bajaj, P. Davies, Measurement of the Poisson's ratio of flexible polyurethane foam and its influence on a uniaxial compression model, Int. J. Eng. Sci. 46 (2008) 31-49.

[29] L.J. Gibson, M.F. Ashby, Cellular Solids. Structure and Properties. Cambridge Solid State Science Series, second ed., Cambridge University Press, 1997.

[30] L.A. Mihai, A. Goriely, Finite deformation effects in cellular structures with hyperelastic cell walls, Int. J. Solid Struct. 53 (2015) 107-128. 